\title{
Synthesis, antibacterial and surface activity of 1,2,4-triazole derivatives
}

\author{
By R. El-Sayed
}

\begin{abstract}
Chemistry Department, Faculty of Science, Benha Unviversity, Benha - Egypt.
\end{abstract} ref_at@ hotmail.com

\section{RESUMEN}

Síntesis y propiedades antibacterianas y tensoactivas de derivados 1,2,4-triazólicos

Es interesante el uso del 1-(4-amino-5-mercapto-4H[1,2,4]triazol-3-il)heptadecano-1-sulfonato sódico (2) como un nuevo precursor en la síntesis de compuestos heterocíclicos biológicamente importantes. La reacción de 2 con disulfuro de carbono en piridina y cloruros de acilo rindió derivados 1,2,4-triazólicos. Condensación de 2 con aldehídos apropiados produjo 5a-c que ciclaron por tratamiento con ácido tioglicólico para dar lugar a 6a-c. Reacciones de 2 con anhídrido ftálico y cloruro de 4-metilbenzenosulfonilo produjeron 7 y $\mathbf{8}$. Además, la reacción de 2 con cloroacetaldehído, bromuro de fenacilo, urea, cloruro de tiacetilo produjo 9,10 , 11, y 12. Por otra parte, el reflujo de 2 con isocianato de fenilo dio 13 y 14 . Todos los productos tuvieron actividad antimicrobiana y propiedades tensoactivas.

PALABRAS-CLAVE: Derivados 1,2,4-triazólicos - Síntesis Propiedades antibacterianas - Propiedades tensoactivas.

\section{SUMMARY}

Synthesis, antibacterial and surface activity of 1,2,4triazole derivatives

The use of sodium 1-(4-amino-5-mercapto-4H-[1,2,4] triazol-3-yl) and heptadecane-1-sulfonate (2) as new precursors to synthesize some important biologically active heterocycles has been found to be effective. The reaction of 2 with carbon disulphide in pyridine and acid chlorides yielded the 1,2,4-triazole derivatives $3, \mathbf{4 a}$ and $\mathbf{4 b}$. The condensation of $\mathbf{2}$ with appropriate aldehydes gave $\mathbf{5 a - c}$ which were cyclized by treating with thioglycollic acid to yield 6a-c. The reactions of 2 with phthalic anhydride and 4methylbenzene-sulfonylchloride gave 7 and 8 . In addition, the reaction of $\mathbf{2}$ with chloroacetaldehyde, phenacyl bromide, urea and chloroacetyl chloride yielded 9, 10, 11 and 12. At the same time, refluxing 2 with phenyl isothiocyanate gave 13 and 14 . All these products have antimicrobial activity and they can be used as surface active agents.

KEY-WORDS: 1,2,4-Triazol derivatives - Synthesis Antibacterial properties - Surface activity properties

\section{INTRODUCTION}

In our series of the synthesis of surface active agents containing heterocyclic moiety (Amin et al., 2004; Amin et al., 2003; El-Sayed et al ., 2005) it is interesting to use 1,2,4-triazole derivatives as new precursor starting material in the synthesis of some important biologically active heterocycles which constitute an important class of organic compounds with diverse biological activities, including antiparastic, analgesic, antibacterial and antiinflammatory activities (Tayseer et al., 2002; Cansiz et al., 2004; Katica et al., 2001; Li-Xue et al., 2002; Hovsepian., 2004; Wasfy., 2003). A considerable effort has been made in recent years to further develop the synthesis of these nucleus. (Boshra et al., 2002; Kumar et al., 2003; Oganisyan et al., 2004). These derivatives are very attractive heterocyclic systems due to their extensive use in medicine, agriculture and industry (Xin-Ping et al., 2000). In light of the above facts and with a view to obtain new biologically active agents I was encouraged to synthesize a new series of 1,2,4triazole derivatives bearing (long alkyl chain with sulfonic acid hydrophilic center) in a single molecular framework likely to constitute new biologically active anionic surface active agent hopefully possessing good surface properties and expected to have biological activities.

\section{MATERIAL AND METHODS}

Melting points are uncorrected. IR spectra in $\mathrm{KBr}$ were measured on a Pye-Uncam SP-1000 infrared spectrophotometer on a $\mathrm{KBr}$ disk or nujol. The ${ }^{1} \mathrm{H}$ NMR spectra were obtained on a Varian EM-390-60 $\mathrm{MHz}$ spectrometer in DMSO as the solvent. Tetramethylsilane TMS served as an internal reference and chemical shifts are expressed as $\delta$ (ppm). Mass spectra were recorded on a G-C/Ms Finning-MAT. Microanalyses were preformed by the Microanalytical Unit at Cairo University. All the compounds gave satisfactory elemental analyses. Thin layer chromatography (TLC) was carried out on silica gel (MN-Kieselgel G., $0.2 \mathrm{~mm}$ thickness) and the plates were scanned under $254 \mathrm{~nm}$ ultraviolet light. Antimicrobial and antifungal activity testes were carried out at the microbiology Lab., Faculty of Science, Zagazig University, Benha-branch, Egypt.

\subsection{Sodium 1-hydrazinocarbonyl- heptadecane-1-sulfonate (1)}

The sodium salt of $\alpha$-sulphonated of stearic acid hydrazide $\mathbf{1}$ was prepared according to the method in the literature (Eissa., 2002). Yield, $70 \%$, 
$\mathrm{mp}=121-122^{\circ} \mathrm{C} . \mathrm{IR}: \mathrm{v}\left(\mathrm{cm}^{-1}\right)=3422,3300(\mathrm{NH})$, 2922-2852 ( $\mathrm{CH}$ in alkyl chain), $1350(\mathrm{~S}=\mathrm{O})$ and $1691(\mathrm{C}=\mathrm{O})$.

\subsection{Sodium 1-(4-amino-5-mercapto-4H-[1,2,4] triazol-3-yl)heptadecane-1-sulfonate (2)}

The acid hydrazide $1(0.01 \mathrm{~mol})$ was added to absolute alcohol $(50 \mathrm{~mL})$, containing $\mathrm{KOH}(1.6 \mathrm{~g})$ at room temperature. Carbon disulphide was added $(2.3 \mathrm{~g}, 0.013 \mathrm{~mol})$ and the mixture stirred at room temperature for $10 \mathrm{~h}$. The mixture was diluted with ether $(30 \mathrm{~mL})$ and stirred for a further $1 \mathrm{~h}$. The potassium salt was used for the next stage without further purification. Hydrazine hydrate (99\%) $(0.02$ mol) was gradually added to the above potassium salt $(0.01 \mathrm{~mol})$ dissolved in water $(20 \mathrm{~mL})$ with stirring and the mixture was refluxed gently for $3 \mathrm{hr}$ during which hydrogen sulphide evolved and the color of the reaction mixture changed to a dark green color, It was then cooled to $5^{\circ} \mathrm{C}$ and acidified with conc. $\mathrm{HCl}$ to $\mathrm{pH} 1.00$. A yellow solid separated out which was filtered, washed with water and crystallized from ethanol to make the triazole 2. Yield, $70 \%, \mathrm{mp}=88-90{ }^{\circ} \mathrm{C}$. IR: $v\left(\mathrm{~cm}^{-1}\right)=3326$ $(\mathrm{NH}), 2920-2850$ (CH in alkyl chain), $2372(\mathrm{SH})$, and $1599 \mathrm{~cm}^{-1}(\mathrm{C}=\mathrm{N}) .{ }^{1} \mathrm{H} \operatorname{NMR}\left(\mathrm{CDCl}_{3}\right): \delta=0.90$ (t, $J=7.2 \mathrm{~Hz}, 3 \mathrm{H}$, terminal $\left.\mathrm{CH}_{3}\right), 1.29-1.33(\mathrm{~m}, 30 \mathrm{H}$, $\mathrm{CH}_{2}$ in alkyl chain), 3.01 (s, $\left.1 \mathrm{H}, \mathrm{SH}\right), 2.0(\mathrm{~s}, 2 \mathrm{H}, \mathrm{NH})$ and 4.25 (t, $\left.1 \mathrm{H}, \mathrm{CH}-\mathrm{SO}_{3} \mathrm{Na}\right)$. Anal. Calcd for $\mathrm{C}_{19} \mathrm{H}_{37} \mathrm{~N}_{4} \mathrm{NaO}_{3} \mathrm{~S}_{2}$ (456.65): C, 49.98; $\mathrm{H}, 8.17 ; \mathrm{N}$, 12.27; S, 14.04. Found C, 50.02; H, 8.13; N, 12.22; S, $14.12 \%$.

\subsection{Sodium 1-(6-thioxo-5,6-dihydro- $[1,2,4]$ triazolo[3,4-b][1,3,4]thiadiazol-3-yl) heptadecane-1-sulfonate (3)}

A mixture of triazole $2(0.01 \mathrm{~mol})$, carbon disulphide $(0.01 \mathrm{~mol})$, and dry pyridine $(20 \mathrm{~mL})$ was heated under reflux for $3 \mathrm{~h}$. It was cooled and poured onto ice/water. A solid product $\mathbf{3}$ was obtained by filtration and crystallization from ethanol. Yield, $73 \%, \mathrm{mp}=62-65^{\circ} \mathrm{C}$. IR: $\mathrm{v} / \mathrm{cm}^{-1}=$ $3229(\mathrm{NH}), 2921-2851(\mathrm{CH}$ in alkyl chain), 1600 $(\mathrm{C}=\mathrm{N})$, and $1375(\mathrm{C}=\mathrm{S}) .{ }^{1} \mathrm{H}$ NMR $\left(\mathrm{CDCl}_{3}\right): \delta=0.96$ (t, $J=7.0 \mathrm{~Hz}, 3 \mathrm{H}$, terminal $\left.\mathrm{CH}_{3}\right), 1.28-1.32(\mathrm{~m}, 30 \mathrm{H}$, $\mathrm{CH}_{2}$ in alkyl chain), $2.0(\mathrm{~s}, 1 \mathrm{H}, \mathrm{NH}$, exchangeable) and $4.23\left(\mathrm{t}, \mathrm{J}=4.7 \mathrm{~Hz}, 1 \mathrm{H}, \mathrm{CH}-\mathrm{SO}_{3} \mathrm{Na}\right)$. MS: $\mathrm{m} / \mathrm{z}$ (\%) $\mathrm{M}^{+}+1=499$ (40). Anal. Calcd for $\mathrm{C}_{20} \mathrm{H}_{35} \mathrm{~N}_{4} \mathrm{NaO}_{3} \mathrm{~S}_{3}$ (498.71): C, 49.17; H, 7.07; N, 11.23; S, 19.29. Found C, 49.12; H, 7.11; N, 11.21 ; S, $19.33 \%$.

\subsection{General procedure for preparation of $4 a$ and $4 b$}

To a solution of triazole $2(0.01 \mathrm{~mol})$ in dry pyridine $(25 \mathrm{~mL})$, the acid chlorides $(0.01 \mathrm{~mol})$, namely, acetyl chloride and/or benzoyl chloride were added in drops. The reaction mixture was stirred at room temperature for $45 \mathrm{~min}$ and then heated for $2 \mathrm{~h}$ in a steam bath. It was then poured onto crushed ice. The solid products obtained by filtration were crystallized from the appropriate solvent to give $\mathbf{4 a}$ and $\mathbf{4 b}$.

2.4.1. Sodium 1-(6-methyl-[1,2,4]triazolo[3,4b][1,3,4]thiadiazol-3-yl)heptadecane-1-sulfonate (4a): Yield, $65 \%$. $\mathrm{mp}=64-66^{\circ} \mathrm{C}$. IR: $\mathrm{v} / \mathrm{cm}^{-1}=2920-$ 2850 ( $\mathrm{CH}$ in alkyl chain), and $1589(\mathrm{C}=\mathrm{N}) .{ }^{1} \mathrm{H}$ NMR $\left(\mathrm{CDCl}_{3}\right): \delta=0.90\left(\mathrm{t}, J=7.0 \mathrm{~Hz}, 3 \mathrm{H}\right.$, terminal $\left.\mathrm{CH}_{3}\right)$, 1.29-1.33 (m, $30 \mathrm{H}, \mathrm{CH}_{2}$ in alkyl chain), 2.35 (s, $\left.J=6.4 \mathrm{~Hz}, 3 \mathrm{H}, \mathrm{CH}_{3}\right), 4.26(\mathrm{t}, J=4.8 \mathrm{~Hz}, 1 \mathrm{H}$, $\mathrm{CH}-\mathrm{SO}_{3} \mathrm{Na}$ ). MS: $\mathrm{m} / \mathrm{z}(\%) \mathrm{M}^{+}=480$ (54). Anal. Calcd for $\mathrm{C}_{21} \mathrm{H}_{37} \mathrm{~N}_{4} \mathrm{NaO}_{3} \mathrm{~S}_{2}$ (480.67): C, 52.48; $\mathrm{H}, 7.76$; N, 11.66; S, 13.34. Found C, 52.44; H, 7.80; N, 11.61; $\mathrm{S}, 13.38 \%$.

2.4.2. Sodium 1-(6-phenyl-[1,2,4]triazolo[3,4b][1,3,4]thiadiazol-3-yl)heptadecane-1-sulfonate (4b). Yield, $75 \%$. $\mathrm{mp}=63-65^{\circ} \mathrm{C}$. IR: $\mathrm{v} / \mathrm{cm}^{-1}=2921-$ 2851 (CH in alkyl chain) and $1599(\mathrm{C}=\mathrm{N}) .{ }^{1} \mathrm{H}$ NMR $\left(\mathrm{CDCl}_{3}\right): \delta=0.95\left(\mathrm{t}, J=7.4 \mathrm{~Hz}, 3 \mathrm{H}\right.$, terminal $\left.\mathrm{CH}_{3}\right)$, 1.28-1.33 (m, $30 \mathrm{H}, \mathrm{CH}_{2}$ in alkyl chain), 4.25 (t, $\left.J=4.4 \mathrm{~Hz}, 1 \mathrm{H}, \mathrm{CH}-\mathrm{SO}_{3} \mathrm{Na}\right)$, and 6.8-7.2 (m, $5 \mathrm{H}$, ArH). Anal. Calcd for $\mathrm{C}_{26} \mathrm{H}_{39} \mathrm{~N}_{4} \mathrm{NaO}_{3} \mathrm{~S}_{2}$ (542.74): C, 57.54; H, 7.24; N, 10.32; S, 11.82. Found C, 57.58; H, 7.34; N, 10.37; S, $11.87 \%$.

\subsection{General procedure for preparation of Schiff bases $5 a-c$}

A mixture of triazole $2(0.01 \mathrm{~mol})$ and the corresponding aldehydes $(0.01 \mathrm{~mol})$ in ethanol $(25$ $\mathrm{mL})$ was treated with concentrated $\mathrm{HCl}(0.5 \mathrm{~mL})$ and refluxed for $2 \mathrm{~h}$. Once cooled, the reaction mixture was filtered and crystallized from ethanol to give 5a-c.

2.5.1. Sodium1-[4-(benzylidene-amino)-5mercarpto-4H-[1,2,4]triazol-3-yl]heptadecane-1sulfonate (5a). Prepared from benzaldehyde. Yield $80 \% . m p=105-107^{\circ} \mathrm{C}$. IR: $v / \mathrm{cm}^{-1}=2920-2850(\mathrm{CH}$ in alkyl chain), $1601(\mathrm{C}=\mathrm{N})$ and $2569(\mathrm{SH})$. The ${ }^{1} \mathrm{H}$ NMR $\left(\mathrm{CDCl}_{3}\right): \delta=0.90(\mathrm{t}, J=7.1 \mathrm{~Hz}, 3 \mathrm{H}$, terminal $\left.\mathrm{CH}_{3}\right), 1.29-1.33\left(\mathrm{~m}, 30 \mathrm{H}, \mathrm{CH}_{2}\right.$ in alkyl chain), 12.86 (s, 1H, SH), 4.27 (t, J=4.0Hz, $\left.1 \mathrm{H}, \mathrm{CH}-\mathrm{SO}_{3} \mathrm{Na}\right)$, 6.9$7.86(\mathrm{~m}, 5 \mathrm{H}, \mathrm{ArH})$ and $8.2(\mathrm{~s}, 1 \mathrm{H}, \mathrm{N}=\mathrm{CH})$. Anal. Calcd for $\mathrm{C}_{26} \mathrm{H}_{41} \mathrm{~N}_{4} \mathrm{NaO}_{3} \mathrm{~S}_{2}$ (544.76): C, 57.33; $\mathrm{H}$, 7.59; N, 10.28; S, 11.77 . Found C, 57.29; H, 7.54; N, 10.24; S, $11.84 \%$.

2.5.2. Sodium1-\{4-[(4-chlorobenzylideneamino]-5-mercarpto-4H-[1,2,4]triazol-3-yl\} heptadecane-1-sulfonate (5b). Prepared from $p$ chlorobenzaldehyde. Yield $80 \% . \mathrm{mp}=110-112{ }^{\circ} \mathrm{C}$. IR: $v / \mathrm{cm}^{-1}=2920-2850(\mathrm{CH}$ in alkyl chain), 1603 $(\mathrm{C}=\mathrm{N})$ and $2537(\mathrm{SH})$. The ${ }^{1} \mathrm{H}$ NMR $\left(\mathrm{CDCl}_{3}\right): \delta=$ 0.95 (t, J=7.0 Hz, 3H, $\left.\mathrm{CH}_{3}\right), 1.27-1.31\left(\mathrm{~m}, 30 \mathrm{H}, \mathrm{CH}_{2}\right.$ in alkyl chain), $4.26\left(\mathrm{t}, \mathrm{J}=3.9 \mathrm{~Hz}, 1 \mathrm{H}, \mathrm{CH}-\mathrm{SO}_{3} \mathrm{Na}\right)$, 6.9-7.4 (m, 4H, ArH) and $8.3(\mathrm{~s}, 1 \mathrm{H}, \mathrm{N}=\mathrm{CH})$. Anal. Calcd for $\mathrm{C}_{26} \mathrm{H}_{40} \mathrm{CIN}_{4} \mathrm{NaO}_{3} \mathrm{~S}_{2}$ (579.20): C, 53.92; $\mathrm{H}$, 6.96; N, 9.67; S, 11.07. Found C, 53.97; H, 7.01; N, $9.72 ;$ S, $11.15 \%$. 
2.5.3. Sodium 1-\{5-mercapto-4[(4-methoxybenzylidene)-amino]-4H-[1,2,4]triazol-3-yl\} heptadecane-1-sulfonate $(5 c)$. Prepared from $p$ methoxybenzaldehyde. Yield $80 \% . \mathrm{mp}=115-117^{\circ} \mathrm{C}$. IR: $v / \mathrm{cm}^{-1}=2920-2850(\mathrm{CH}$ in alkyl chain), 1605 $(\mathrm{C}=\mathrm{N})$ and $2557(\mathrm{SH})$. The ${ }^{1} \mathrm{H}$ NMR $\left(\mathrm{CDCl}_{3}\right): \delta=0.90$ (t, $3 \mathrm{H}$, terminal $\left.\mathrm{CH}_{3}\right), 1.29-1.34\left(\mathrm{~m}, 30 \mathrm{H}, \mathrm{CH}_{2}\right.$ in alkyl chain), $3.73\left(\mathrm{~s}, 3 \mathrm{H}, \mathrm{OCH}_{3}\right), 4.22(\mathrm{t}, 1 \mathrm{H}, \mathrm{CH}-$ $\left.\mathrm{SO}_{3} \mathrm{Na}\right)$, 6.9-7.4 (m, 4H, ArH). Anal. Calcd for $\mathrm{C}_{27} \mathrm{H}_{43} \mathrm{~N}_{4} \mathrm{NaO}_{4} \mathrm{~S}_{2}$ (574.79): C, 56.42; $\mathrm{H}, 7.54 ; \mathrm{N}$, 9.75; S, 11.16. Found C, 56.48; H, 7.61; N, 9.70; S, $10.99 \%$.

\subsection{General procedure for preparation of $6 a-c$}

To a solution of Schiff bases 5a-c $(0.01 \mathrm{~mol})$ dry acetone thioglycollic acid $(0.01 \mathrm{~mol})$ was added. The reaction mixture was refluxed for $3 \mathrm{~h}$. A solid product was obtained after cooling to give the adducts $6 a-c$ which were crystallized from ethanol.

2.6.1. Sodium1-[5-mercapto-4[(4-oxo-2-phenyIthiazolidin-3-yl)-4H-[1,2,4]triazol-3-yl] heptadecane-1-sulfonate (6a). Yield 76\%. $\mathrm{mp}=78$ $80^{\circ} \mathrm{C}$. IR: $v / \mathrm{cm}^{-1}=2919-2851(\mathrm{CH}$ in alkyl chain), $2560(\mathrm{SH}) 1593(\mathrm{C}=\mathrm{N}) .{ }^{1} \mathrm{H}$ NMR $\left(\mathrm{CDCl}_{3}\right): \delta=0.96$ (t, $J=7.4 \mathrm{~Hz}, 3 \mathrm{H}$, terminal $\left.\mathrm{CH}_{3}\right), 1.28-1.33(\mathrm{~m}, 30 \mathrm{H}$, $\mathrm{CH}_{2}$ in alkyl chain), $3.02(\mathrm{~s}, 1 \mathrm{H}, \mathrm{SH}), 3.27,3.38$ (2s, $2 \mathrm{H}, \mathrm{CH}_{2}$ of the ring), 4.27 (t, $J=4.2 \mathrm{~Hz}, 1 \mathrm{H}, \mathrm{CH}-$ $\left.\mathrm{SO}_{3} \mathrm{Na}\right), 5.92(\mathrm{~s}, 1 \mathrm{H}, \mathrm{CH}-\mathrm{Ph})$ and 7.06-7.14 $(\mathrm{m}, 5 \mathrm{H}$, ArH). Anal. Calcd for $\mathrm{C}_{28} \mathrm{H}_{43} \mathrm{~N}_{4} \mathrm{NaO}_{4} \mathrm{~S}_{3}$ (618.86): C, 54.34; H, 7.00; N, 9.05; S, 15.54. Found C, 54.41; H, $7.11 ; \mathrm{N}, 9.12 ; \mathrm{S}, 15.48 \%$.

2.6.2. Sodium1-\{4-[2-(4-chlorophenyl)-4-oxothiazolidin-3-yl]-5-mercarpto-4H-[1,2,4]triazol-3-yl\} hept-adecane-1-sulfonate (6b). Yield $66 \% . \mathrm{mp}=83$ $85^{\circ} \mathrm{C}$. IR: $\mathrm{v} / \mathrm{cm}^{-1}=2920-2850(\mathrm{CH}$ in alkyl chain), $2527(\mathrm{SH}), 1600(\mathrm{C}=\mathrm{N}) .{ }^{1} \mathrm{H}$ NMR $\left(\mathrm{CDCl}_{3}\right): \delta=0.90$ (t, $J=7.1 \mathrm{~Hz}, 3 \mathrm{H}$, terminal $\left.\mathrm{CH}_{3}\right), 1.27-1.31(\mathrm{~m}, 30 \mathrm{H}$, $\mathrm{CH}_{2}$ in alkyl chain), $3.0(\mathrm{~s}, 1 \mathrm{H}, \mathrm{SH}), 4.26$ (t, $J=4.3 \mathrm{~Hz}$, $\left.1 \mathrm{H}, \mathrm{CH}-\mathrm{SO}_{3} \mathrm{Na}\right), 3.26,3.31\left(2 \mathrm{~s}, 2 \mathrm{H}, \mathrm{CH}_{2}\right.$ of the ring) $5.12(\mathrm{~s}, 1 \mathrm{H}, \mathrm{CH}-\mathrm{Ph})$ and 6.3-7.5 (m, $4 \mathrm{H}, \mathrm{ArH}) . \mathrm{MS}$ : $\mathrm{m} / \mathrm{z}(\%) \mathrm{M}^{+}+1=654$ (33). Anal. Calcd for $\mathrm{C}_{28} \mathrm{H}_{42} \mathrm{ClN}_{4} \mathrm{NaO}_{4} \mathrm{~S}_{3}$ (653.31): C, 51.48; $\mathrm{H}, 6.48 ; \mathrm{N}$, 8.58; S, 14.72. Found $\mathrm{C}, 51.54 ; \mathrm{H}, 6.45 ; \mathrm{N}, 8.52 ; \mathrm{S}$, $14.78 \%$.

2.6.3. Sodium 1-\{5-mercarpto-4-[2-(4methoxyphenyl)-4-oxothiazolidin-3-yl]-4H[1,2,4]triazol-3-yl\}hep-tadecane-1-sulfonate (6c). Yield $60 \%$. $\mathrm{mp}=81-83^{\circ} \mathrm{C}$. IR: $\mathrm{v} / \mathrm{cm}^{-1}=2920-2850$ (CH in alkyl chain), $2520(\mathrm{SH}), 1599(\mathrm{C}=\mathrm{N}) .{ }^{1} \mathrm{H}$ NMR $\left(\mathrm{CDCl}_{3}\right): \delta=0.95\left(\mathrm{t}, 3 \mathrm{H}\right.$, terminal $\left.\mathrm{CH}_{3}\right), 1.1(\mathrm{~s}, 1 \mathrm{H}$, $\left.\mathrm{OCH}_{3}\right), 1.3-1.5\left(\mathrm{~m}, 30 \mathrm{H}, \mathrm{CH}_{2}\right.$ of alkyl chain), 3.1 (s, $1 \mathrm{H}, \mathrm{SH}), 4.26\left(\mathrm{t}, 1 \mathrm{H}, \mathrm{CH}-\mathrm{SO}_{3} \mathrm{Na}\right), 3.35\left(\mathrm{~s}, 2 \mathrm{H}, \mathrm{CH}_{2}\right.$ of the ring), $4.01\left(\mathrm{~s}, 3 \mathrm{H}, \mathrm{OCH}_{3}\right), 5.23(\mathrm{~s}, 1 \mathrm{H}, \mathrm{CH}-\mathrm{Ph})$ and 6.5-7.2 (m, $4 \mathrm{H}$, ArH). Anal. Calcd for $\mathrm{C}_{29} \mathrm{H}_{45} \mathrm{~N}_{4} \mathrm{NaO}_{5} \mathrm{~S}_{3}$ (648.89): C, 53.68; $\mathrm{H}, 6.99 ; \mathrm{N}$, 8.63; S, 14.82. Found C, 53.72; H, 7.11; N, 8.68; S, $14.86 \%$.

\subsection{Sodium1-[4-(1,3-dioxo-1,3-dihydroisoindol- 2-yl)-5-mercapto-4H-[1,2,4]triazol-3-yl] heptadecane-1-sulfonate (7).}

A mixture of triazole $2(0.01 \mathrm{~mol})$ and phthalic anhydride $(0.01 \mathrm{~mol})$, in butanol $(20 \mathrm{~mL})$ was heated under reflux for $4 \mathrm{~h}$. Then the solution was concentrated. A solid product 7 was obtained by filtration which was crystallized from ethanol. Yield 55 $\% . \mathrm{mp}=91-93^{\circ} \mathrm{C}$. IR: $\mathrm{v} / \mathrm{cm}^{-1}=2920-2850(\mathrm{CH}$ in alkyl chain), 3047 (CH aromatic), $2461(\mathrm{SH}), 1695,1988$ $(\mathrm{C}=\mathrm{O})$ and $1599(\mathrm{C}=\mathrm{N}) .{ }^{1} \mathrm{H}$ NMR $\left(\mathrm{CDCl}_{3}\right): \delta=0.83(\mathrm{t}$, $3 \mathrm{H}$, terminal $\left.\mathrm{CH}_{3}\right), 1.29-1.33\left(\mathrm{~m}, 30 \mathrm{H}, \mathrm{CH}_{2}\right.$ in alkyl chain), $3.01(\mathrm{~s}, 1 \mathrm{H}, \mathrm{SH}), 4.27\left(\mathrm{t}, 1 \mathrm{H}, \mathrm{CH}-\mathrm{SO}_{3} \mathrm{Na}\right)$ and 8.2-8.7 (m, $4 \mathrm{H}$, ArH). Anal. Calcd for $\mathrm{C}_{27} \mathrm{H}_{39} \mathrm{~N}_{4} \mathrm{NaO}_{5} \mathrm{~S}_{2}$ (586.75): C, 55.27; H, 6.70; N, 9.55; S, 10.93. Found C, $55.21 ; \mathrm{H}, 6.76 ; \mathrm{N}, 9.61 ; \mathrm{S}, 10.86 \%$.

\subsection{Sodium 1[5-mercarpto-4-(toluene-4- sulfonylamino)-4H-[1,2,4]triazol-3-yl) heptadecane-1-sulfonate (8).}

A mixture of triazole $2(0.01 \mathrm{~mol})$ and 4methylbenzene sulfonylchloride $(0.01 \mathrm{~mol})$ in dry pyridine $(20 \mathrm{~mL})$ was heated under reflux for $3 \mathrm{~h}$. It was then cooled and poured onto ice/water. A solid product 8 was obtained by filtration which was crystallized from ethanol. Yield, $73 \%, \mathrm{mp}=73-75$ ${ }^{\circ} \mathrm{C}$. IR: $v / \mathrm{cm}^{-1}=3250(\mathrm{NH}), 2921-2851(\mathrm{CH}$ in alkyl chain), $1610(\mathrm{C}=\mathrm{N}), 1170$ and $944\left(\mathrm{SO}_{2}\right)$ and 2455 (SH). ${ }^{1} \mathrm{H}$ NMR $\left(\mathrm{CDCl}_{3}\right): \delta=0.95(\mathrm{t}, 3 \mathrm{H}$, terminal $\left.\mathrm{CH}_{3}\right), 1.27-1.31\left(\mathrm{~m}, 30 \mathrm{H}, \mathrm{CH}_{2}\right.$ in alkyl chain), 2.0 (s, $1 \mathrm{H}, \mathrm{NH}$, exchangeable), 2.35 (s, 3H, Ph- $\left.\mathrm{CH}_{3}\right), 3.1$ (s, $1 \mathrm{H}, \mathrm{SH}), 4.27\left(\mathrm{t}, 1 \mathrm{H}, \mathrm{CH}-\mathrm{SO}_{3} \mathrm{Na}\right.$ ) and 7.34-7.81 $(\mathrm{m}, 4 \mathrm{H}, \mathrm{ArH})$. Anal. Calcd for $\mathrm{C}_{26} \mathrm{H}_{43} \mathrm{~N}_{4} \mathrm{NaO}_{5} \mathrm{~S}_{3}$ (610.84): C, 51.12; H, 7.10; N, 9.17; S, 15.75. Found C, $51.06 ; \mathrm{H}, 7.15 ; \mathrm{N}, 9.11 ; \mathrm{S}, 15.78 \%$.

\subsection{Sodium 1-(7H-[1,2,4]triazol[3,4-b][1,3,4] thiadiazin-3-yl)heptadecane-1-sulfonate (9)}

A mixture of triazole $2(0.01 \mathrm{~mol})$, chloroacetaldehyde $(0.02 \mathrm{~mol})$ and conc. $\mathrm{HCl}$ $(2 \mathrm{~mL})$ in ethanol $(50 \mathrm{~mL})$ was refluxed for $3 \mathrm{~h}$. After the removal of ethanol under reduced pressure, the resulting solid was filtered and washed with water. The crude product was crystallized from ethanol to give 9. Yield $73 \% . \mathrm{mp}=68-70^{\circ} \mathrm{C}$. IR: $\mathrm{v} / \mathrm{cm}^{-1}=2920-$ $2850\left(\mathrm{CH}\right.$ in alkyl chain), $1610(\mathrm{C}=\mathrm{N}) .{ }^{1} \mathrm{H}$ NMR $\left(\mathrm{CDCl}_{3}\right): \delta=0.90\left(\mathrm{t}, 3 \mathrm{H}\right.$, terminal $\left.\mathrm{CH}_{3}\right), 1.29-1.33$ (m, $30 \mathrm{H}, \mathrm{CH}_{2}$ in alkyl chain), $4.23(\mathrm{t}, 1 \mathrm{H}, \mathrm{CH}-$ $\left.\mathrm{SO}_{3} \mathrm{Na}\right), 3.1\left(\mathrm{~d}, J=4 \mathrm{~Hz}, 2 \mathrm{H}, \mathrm{CH}_{2}\right.$ of the ring) and $7.5(\mathrm{t}, 1 \mathrm{H},=\mathrm{CH})$. Anal. Calcd for $\mathrm{C}_{21} \mathrm{H}_{37} \mathrm{~N}_{4} \mathrm{NaO}_{3} \mathrm{~S}_{2}$ (480.67): C, 52.48; H, 7.76; N, 11.66; S, 13.34. Found C, 52.53; H, 7.81; N, 11.71; S, $13.27 \%$.

\subsection{Sodium 1-(6-phenyl-7H-[1,2,4]triazolo [3,4-b][1,3,4]thiadiazin-3-yl) heptadecane-1-sulfonate (10)}

A suspension of triazole $2(0.1 \mathrm{~mol})$ and phenacyl bromide $(0.13 \mathrm{~mol})$ in absolute ethanol $(25 \mathrm{~mL})$ was heated under reflux for $3 \mathrm{~h}$, then $(0.01$ 
mol) of anhydrous sodium acetate was added. The reaction mixture was heated for an additional $1 \mathrm{~h}$, then cooled and poured onto ice-cold water. The solid product was crystallized from ethanol to make 10. Yield $67 \%$; $\mathrm{mp}=77-79{ }^{\circ} \mathrm{C}$. IR: $\mathrm{v} / \mathrm{cm}^{-1}=2910$ $2850(\mathrm{CH}$ in alkyl chain), $1589(\mathrm{C}=\mathrm{N})$ and 1349 $(\mathrm{C}=\mathrm{S}) .{ }^{1} \mathrm{H}$ NMR $\left(\mathrm{CDCl}_{3}\right): \delta=0.96(\mathrm{t}, J=7.0 \mathrm{~Hz}, 3 \mathrm{H}$, terminal $\left.\mathrm{CH}_{3}\right)$, 1.29-1.33 $\left(\mathrm{m}, 30 \mathrm{H}, \mathrm{CH}_{2}\right.$ in alkyl chain), $3.0\left(\mathrm{~s}, 2 \mathrm{H}, \mathrm{CH}_{2}\right.$ of the ring), 4.27 (t, $J=4.2 \mathrm{~Hz}$, $\left.1 \mathrm{H}, \mathrm{CH}-\mathrm{SO}_{3} \mathrm{Na}\right)$, and 6.3-7.5 (m, $\left.5 \mathrm{H}, \mathrm{ArH}\right)$. Anal. Calcd for $\mathrm{C}_{27} \mathrm{H}_{41} \mathrm{~N}_{4} \mathrm{NaO}_{3} \mathrm{~S}_{2}$ (556.25): C, 58.25; $\mathrm{H}$, 7.42; N, 10.06; S, 11.52. Found C, 58.19; H, 7.37; N, $10.01 ;$ S, $11.45 \%$.

\subsection{Sodium 1-(6-oxo-5,6-dihydro-[1,2,4] triazolo[3,4-b][1,3,4]thiadiazol-3-yl) heptadecane-1-sulfonate (11)}

A mixture of triazole $2(0.1 \mathrm{~mol})$ and urea $(0.13$ mol) was heated at $180-190{ }^{\circ} \mathrm{C}$ for $6 \mathrm{~h}$. The reaction mixture was cooled and added to a solution of sodium hydroxide $(5 \%, 20 \mathrm{ml})$, then filtered and the filtrate was acidified with dilute $\mathrm{HCl}$. The solid product was crystallized from ethanol to give 11 . Yield, $81 \%$; mp 76-78 ${ }^{\circ} \mathrm{C}$, IR: $v / \mathrm{cm}^{-1}=3228(\mathrm{NH})$, 2921-2852 (CH aliphatic), 1675 (CO), 1598 (C=N). ${ }^{1} \mathrm{H}$ NMR $\left(\mathrm{CDCl}_{3}\right): \delta=0.90(\mathrm{t}, J=7.2 \mathrm{~Hz}, 3 \mathrm{H}$, terminal $\left.\mathrm{CH}_{3}\right), 1.28-1.33\left(\mathrm{~m}, 30 \mathrm{H}, \mathrm{CH}_{2}\right.$ in alkyl chain), 4.24 (t, $\left.J=3.9 \mathrm{~Hz}, 1 \mathrm{H}, \mathrm{CH}-\mathrm{SO}_{3} \mathrm{Na}\right)$ and 8.0 (s, H, NH, exchangeable). MS $\mathrm{m} / \mathrm{z}(\%): \mathrm{M}^{+}=482$ (45). Anal. Calcd for $\mathrm{C}_{20} \mathrm{H}_{35} \mathrm{~N}_{4} \mathrm{O}_{4} \mathrm{~S}_{2}$ (482.64): C, 49.77; H, 7.31; N, 11.61; S, 13.95. Found: C, 49.82; H, 7.35; N, 11.66; S, $13.87 \%$.

\subsection{Sodium 1-(7-oxo-6,7-dihydro-5H-[1,2,4] triazolo[3,4-b][1,3,4]thiadiazol-3-yl) heptadecane-1-sulfonate (12)}

A mixture of triazole $2(0.1 \mathrm{~mol})$ and chloroacetyl chloride $(0.1 \mathrm{~mol})$ in dry dioxane $(30 \mathrm{~mL})$ was left to stand at room temperature overnight. The precipitated solid was filtered off and crystallized from benzene to give 12. Yield $70 \% . m p=60-62{ }^{\circ} \mathrm{C}$. IR: $v / \mathrm{cm}^{-1}=3341(\mathrm{NH}), 2921-2850$ (CH aliphatic), $1676(\mathrm{CO})$, and $1587(\mathrm{C}=\mathrm{N}) .{ }^{1} \mathrm{H} \mathrm{NMR}\left(\mathrm{CDCl}_{3}\right)$ : $\delta=0.95\left(\mathrm{t}, \mathrm{J}=7.5 \mathrm{~Hz}, 3 \mathrm{H}\right.$, terminal $\mathrm{CH}_{3}$ ), 1.27-1.35 (m, $30 \mathrm{H}, \mathrm{CH}_{2}$ in alkyl chain), 4.22 (t, $J=4.5 \mathrm{~Hz}, 1 \mathrm{H}$, $\left.\mathrm{CH}-\mathrm{SO}_{3} \mathrm{Na}\right), 3.72\left(\mathrm{~s}, 2 \mathrm{H}, \mathrm{CH}_{2}\right.$ of the ring) and 2.1 (s, $\mathrm{H}, \mathrm{NH}$, which is exchangeable). $\mathrm{MS}: \mathrm{m} / \mathrm{z}(\%) \mathrm{M}^{+}=$ 496 (69). Anal. Calcd for $\mathrm{C}_{21} \mathrm{H}_{37} \mathrm{~N}_{4} \mathrm{NaO}_{4} \mathrm{~S}_{2}$ (496.67): C, 50.78; H, 7.51; N, 11.28; S, 12.91. Found C, 50.73; H, 7.45; N, 11.21; S, $12.97 \%$.

\subsection{Sodium 1-[5-mercapto-4- (3-phenylthioureido)-4H-[1,2,4]triazol-3-yl] heptadecane-1-sulfonate (13)}

A mixture of triazole $2(0.1 \mathrm{~mol})$, phenyl isothiocyanate $(0.1 \mathrm{~mol})$ and powdered sodium hydroxide $(0.8 \mathrm{~g})$ in DMF $(25 \mathrm{~mL})$ was stirred at room temperature for $24 \mathrm{~h}$. The reaction mixture was poured onto dilute acetic acid $(5 \%, 15 \mathrm{~mL})$. The precipitated product was filtered and crystallized from ethanol to give 13 . Yield $85 \% ; m p=108{ }^{\circ} \mathrm{C}$, IR: $v / \mathrm{cm}^{-1}=3250,3246(\mathrm{NH}), 2546(\mathrm{SH})$ and 1376 $(\mathrm{C}=\mathrm{S}) .{ }^{1} \mathrm{H}$ NMR $\left(\mathrm{CDCl}_{3}\right): \delta=0.90(\mathrm{t}, \mathrm{J}=7.6 \mathrm{~Hz}, 3 \mathrm{H}$, terminal $\left.\mathrm{CH}_{3}\right)$, 1.29-1.34 $\left(\mathrm{m}, 30 \mathrm{H}, \mathrm{CH}_{2}\right.$ in alkyl chain), $2.0,4.0(\mathrm{~s}, 2 \mathrm{H}, 2 \mathrm{NH}), 13.01(\mathrm{~s}, 1 \mathrm{H}, \mathrm{SH})$, $4.25\left(\mathrm{t}, 1 \mathrm{H}, \mathrm{CH}-\mathrm{SO}_{3} \mathrm{Na}\right)$ and 6.41-7.1 (m, $\left.5 \mathrm{H}, \mathrm{ArH}\right)$. Anal. Calcd for $\mathrm{C}_{26} \mathrm{H}_{42} \mathrm{~N}_{5} \mathrm{NaO}_{3} \mathrm{~S}_{3}$ (591.84): C, 52.77; H, 7.15; N, 11.83; S, 16.25. Found C, 52.71; H, 7.08; N, $11.77 ;$ S, $16.31 \%$.

\subsection{Sodium 1-(6-phenylamino-[1,2,4] triazolo[3,4-b][1,3,4]thiadiazol-3-yl) heptadecane-1-sulfonate (14)}

Methode A. A mixture of phenyl isothiocyanate $(0.1 \mathrm{~mol})$, triazole 2 ( $0.1 \mathrm{~mol})$ and powdered sodium hydroxide $(0.8 \mathrm{~g})$ in DMF $(25 \mathrm{~mL})$ was refluxed for $4 \mathrm{~h}$. The reaction mixture was poured onto dilute acetic acid $(5 \%, 15 \mathrm{~mL})$. The precipitated product was filtered and crystallized from ethanol to give 14 . Yield $78 \%$; $m p=85-87^{\circ} \mathrm{C}$. IR: $v / \mathrm{cm}^{-1}=3270(\mathrm{NH})$ and $1590(\mathrm{C}=\mathrm{N}) .{ }^{1} \mathrm{H}$ NMR $\left(\mathrm{CDCl}_{3}\right): \delta=0.95(\mathrm{t}, 3 \mathrm{H}$, terminal $\left.\mathrm{CH}_{3}\right), 1.28-1.33\left(\mathrm{~m}, 30 \mathrm{H}, \mathrm{CH}_{2}\right.$ in alkyl chain), $4.24\left(\mathrm{t}, 1 \mathrm{H}, \mathrm{CH}-\mathrm{SO}_{3} \mathrm{Na}\right), 4.0(\mathrm{~s}, \mathrm{H}, \mathrm{NH}$, exchangeable) and 6.46-7.2 (m, $5 \mathrm{H}, \mathrm{ArH})$. Anal. Calcd for $\mathrm{C}_{26} \mathrm{H}_{40} \mathrm{~N}_{5} \mathrm{NaO}_{3} \mathrm{~S}_{2}$ (557.76): C, 55.99; $\mathrm{H}$, 7.23; N, 12.56; S, 11.50. Found C, 56.05; H, 7.28; N, 12.62; S, $11.57 \%$.

Methode $B$. Thiosemicarbazide derivative 13 was fused in an oil-bath above its melting point. The product was cooled, treated with ethyl acetate, and filtered. The solid product was crystallized from ethanol to give 14 .

\subsection{Biological activity}

The antibacterial activities of some synthesized compounds were determined in vitro using hole plate and filter paper disc methods (Eissa et al., 2003) against various pathogenic bacteria such as Gram +ve bacteria (Bacillus subtilis, staphylococcus aureus) and gram -ve bacteria (Escher-ichia coli) in addition to fungi as Aspergillus niger was used. The tested compounds were dissolved in $10 \%$ acetone $(\mathrm{v} / \mathrm{v})$, different concentrations were chosen (125, $250,500 \mu \mathrm{g} / \mathrm{ml}$ ). A qualitative screen was performed on all compounds while quantitative assays were done on active compounds only.

\subsection{Surface active properties}

2.16.1. Surface and interfacial tension. Both surface and interfacial tension were measured using Du-Nouy tensiometer (Findly., 1963) (Kruss, Type 8451) with $0.1 \mathrm{wt} \%$ aqueous solution at room temperature $\left(25^{\circ} \mathrm{C}\right)$.

2.16.2. Kraft point. The Kraft point of the prepared anionic surfactants was measured as the temperature at which $1.0 \%$ solution becomes clear under gradual heating (Wiel et al., 1963). 
2.16.3. Wetting time. The wetting time was determined by immersing a sample of cotton fabric in 1.0 wt \% aqueous solution of surfactants. (Masuyama et al., 1978).

2.16.4. Foaming properties. The foaming properties were measured according to Somaya et al. (1998). In this procedure a $25 \mathrm{ml}$ solution (1.0 wt $\%$ ) was shaken vigorously for 10 seconds in a 100 $\mathrm{ml}$ glass stopper, graduated cylinder, at $25^{\circ} \mathrm{C}$. The solution was allowed to stand for 30 seconds, and the foam height was measured.

2.16.5. Emulsification stability. The emulsion was prepared from $10 \mathrm{ml}$.of a $20 \mathrm{~m}$ mol. aqueous solution of surfactant and $5 \mathrm{ml}$. of toluene at $40 \mathrm{C}$. The emulsifying property was determined by the time it took for an aqueous volume separating from the emulsion layer to reach $9 \mathrm{ml}$. counting from the moment of cession shaking (Takeshi, 1970).

2.16.6. Stability towards hydrolysis. A mixture of 10 m.mol surfactant and $10 \mathrm{ml} 0.05 \mathrm{~N} \mathrm{NaOH}$ were placed in a thermostat at $40^{\circ} \mathrm{C}$. The time it takes a sample solution to be clouded as a result of hydrolysis shows the stability of the surfactant to hydrolysis (El-Sukkary et al., 1987).

\subsection{Biodegradability}

Samples taken daily or more frequently were filtered through Wattmann filter paper number (1) before measuring the surface tension. Surface tension measurements were made periodically each day, on each sample during the degradation test (Eter et al., 1974). Biodegradation percent (D) for each sample was calculated using the following equation: $D=\left[\begin{array}{ll}\left(\gamma_{t}-\gamma_{0}\right) & \left(\gamma_{b t}-\gamma_{0}\right)\end{array}\right] \times 100$, where $\gamma_{t}=$ surface tension at time $t, \gamma_{0}=$ surface tension at zero time, $\gamma_{\mathrm{bt}}=$ surface tension of blank experiment at time $\mathrm{t}$ (without samples).

\section{RESULTS AND DISCUSSION}

\subsection{Synthesis}

In the present work, the reaction of sodium salt of $\alpha$-sulphonated fatty acid hydrazide 1 with carbon disulphide in ethanolic potassium hydroxide gave the potassium salt of the corresponding dithiocarbazate in quantitive yeild. Furthermore, the potassium salts upon reaction with hydrazine hydrate (99\%) gave sodium1-(4-amino-5-mercapto-4H-[1,2,4]triazol-3yl)-heptade-cane-1-sulfonate (2), which required a starting material.(Scheme 1)

The triazole 2 treated with carbon disulfide in pyridine afforded sodium 1-(6-thioxo-5,6-dihydro$[1,2,4]$ triazolo[3,4-b][1,3,4]thiadiazol-3-yl) heptadecane-1-sulfonate (3). Compounds $4 a$ and $\mathbf{4 b}$ were obtained through the reaction of triazole 2 with acid chlorides (acetyl chloride and benzoyl chloride). Condensation of triazole 2 with aromatic aldehydes (benzaldehyde, p-chloro-benzaldehyde

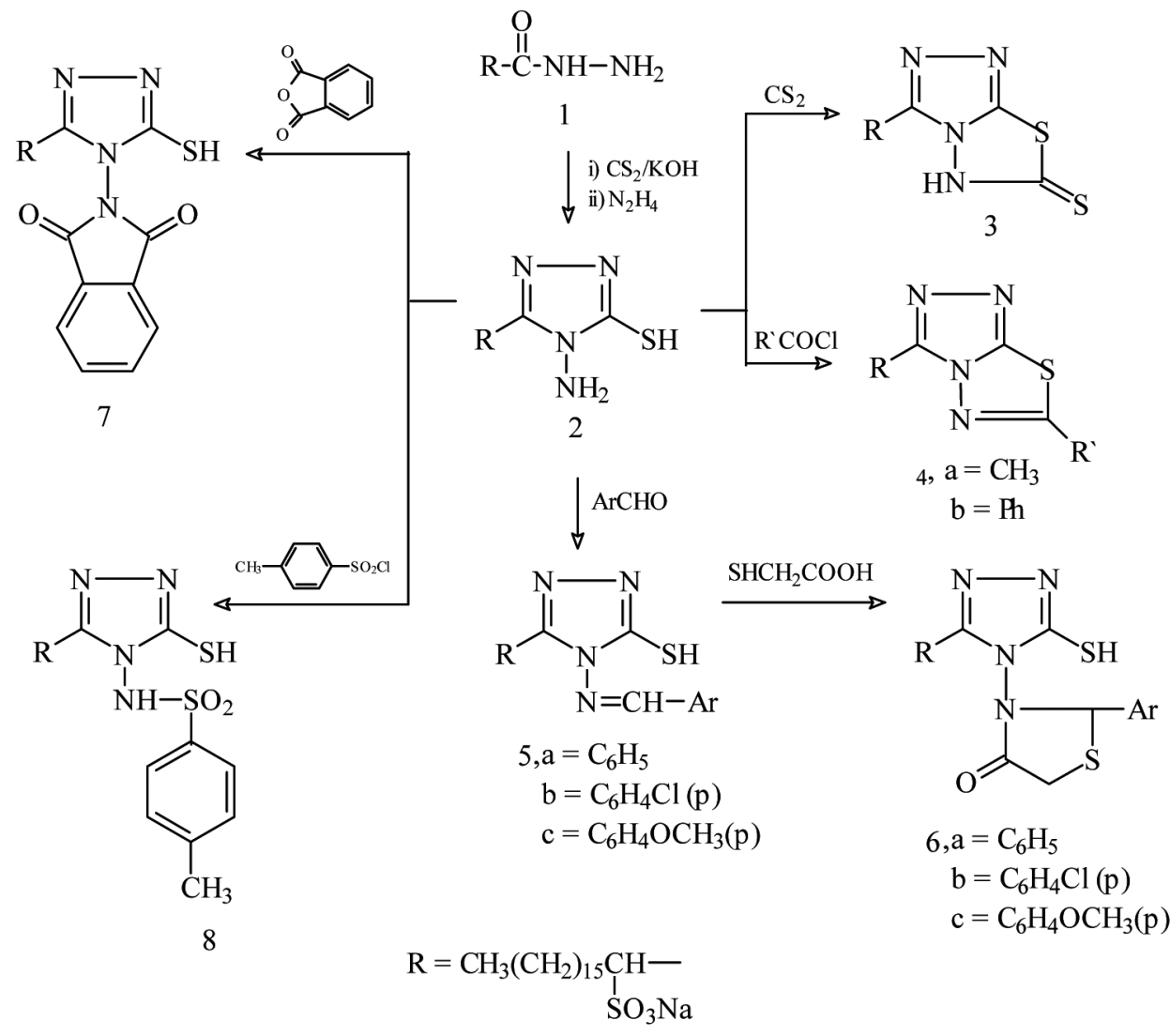

Scheme 1 
and $p$-methoxybenzaldehyde) in refluxing ethanol containing catalytic amounts of piperidine furnished the Schiff bases $\mathbf{5 a - c}$. Also, the reactivity of $\mathbf{5 a - c}$ towards other reagents has been investigated to obtain newer biologically active heterocycles system. Thus, the reaction of Schiff base 5a-c with thioglycollic acid gave 6 a-c. The reaction of triazole 2 with phthalic anhydride in butanol gave 7 . The addition of 4-methylbenzene sulfonylchloride to triazole 2 gave $\mathbf{8}$. On the other hand, the reaction of triazole 2 with one equivalent of chloroacetaldehyde in refluxing ethanol produced sodium $1-(7 \mathrm{H}-$ $[1,2,4]$ triazolo[3,4-b][1,3,4]thiadiazin-3-yl)heptadec-ane-1-sulfonate (9).

As anticipated, the condensation of 2 with equimolar amounts of phenacyl bromides in the presence of potassium carbonate in absolute ethanol resulted in cyclocondensation to give the corresponding sodium-1-[6-phenyl-7H-[1,2,4]triazolo [3,4-b][1,3,4]thiadiazin-3-yl]-heptadecane -1-sulfonate (10). The fusion of triazole 2 with urea gave $\mathbf{1 1}$ in good yield (80\%).

In the present investigation, the condensation of 2 with equimolar amounts of chloroacetyl chloride furnished 12. In view of the known antifungal and antiviral characteristics (Walid et al., 2002; Stankovsk et al., 2000) inherent in substituted thiosemicarbazide derivatives, the synthesis of new compounds incorporating such a group was undertaken. Thus, the reaction of triazole 2 with phenyl isothiocyanate in dimethylformamide at room temperature gave 13. On the other hand, the reaction of triazole 2 with phenyl isothiocyanate by refluxing in dimethylformamide resulted in the corresponding thiosemicarbazide derivative 14 which was also obtained by heating 13 above its melting point. (Scheme 2).

\subsection{Biological activity}

The data indicated that most of the synthesized compounds have remarkable activity and that the tested compounds 2, 6a-c, 10, 9, 12 and $\mathbf{1 3}$ were highly active towards the selected pathogens, while the compounds $3,4 a, 4 b, 7 a-c, 8$, and 11 were moderately active towards the different strains of bacteria and fungi as compared with the standard. (Table I).

\subsection{Surface active properties}

The investigation of the surface active properties (surface and interfacial tension, Kraft point, wetting time, foaming height, emulsion stability, and stability against hydrolysis) of 1,2,4-triazole derivatives bearing (long alkyl chain with sulfonic acid hydrophilic center) was carried out at concentration $1 \mathrm{wt} \%$ and $20^{\circ} \mathrm{C}$ in distilled water. The results are represented in Table II. The biodegradability properties were also determined and are represented in Table III. These products are interesting because these types of anionic surfactants are not common ones. Therefore the traditional procedure was used to follow up the properties.

3.3.1. Surface and interfacial tension. The results indicate that all the synthesized products have pronounced surface activity. Where the values

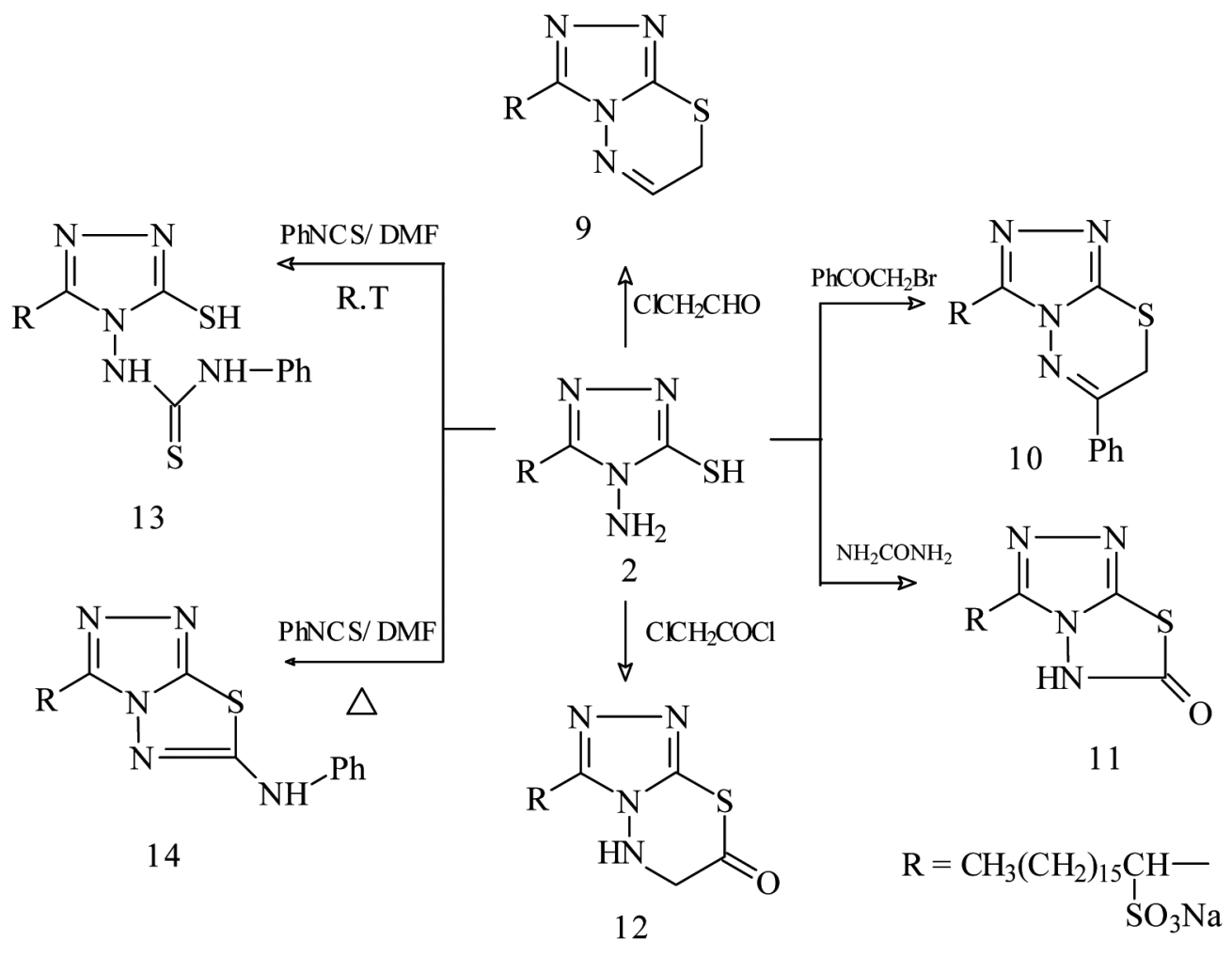

Scheme 2 
Table I

The antimicrobial activity of the tested compounds

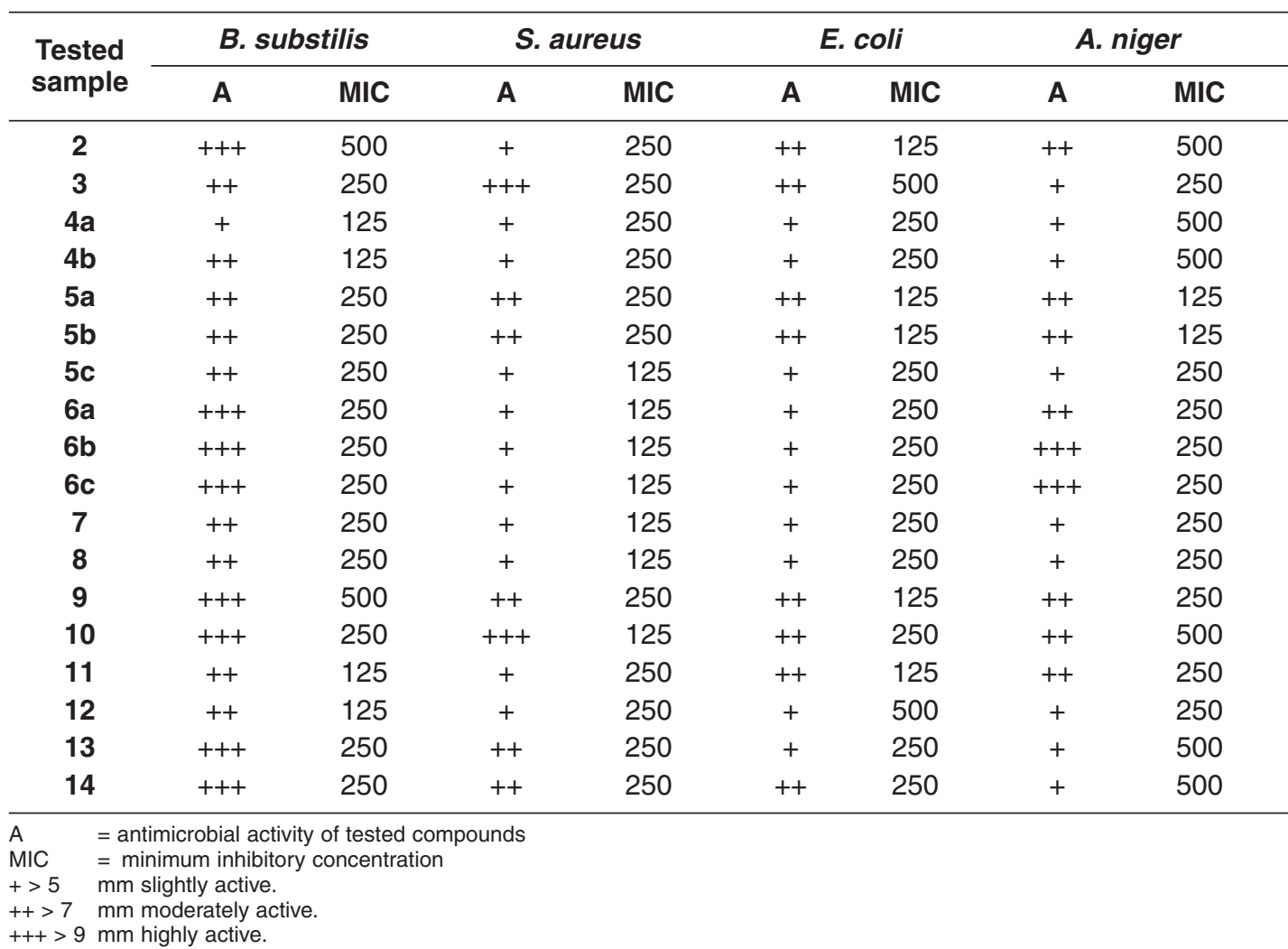

Table II

Surface properties of the synthesized compounds

\begin{tabular}{cccccccc}
\hline $\begin{array}{c}\text { Compd } \\
\text { No. }\end{array}$ & $\begin{array}{c}\text { Surface } \\
\text { tension } \\
\text { (dyne/cm) } \\
\mathbf{0 . 1} \mathbf{~ m / l}\end{array}$ & $\begin{array}{c}\text { Interfacial } \\
\text { tension } \\
\text { (dyne/cm) } \\
\mathbf{0 . 1} \mathbf{~ m / l}\end{array}$ & $\begin{array}{c}\text { Kraft } \\
\text { Point } \\
{ }^{\circ} \mathbf{C}\end{array}$ & $\begin{array}{c}\text { Wetting } \\
\text { time } \\
\text { (sec.) }\end{array}$ & $\begin{array}{c}\text { Emulsion } \\
\text { stability } \\
\text { (min:sec) }\end{array}$ & $\begin{array}{c}\text { Foam } \\
\text { height } \\
\text { (mm) }\end{array}$ & $\begin{array}{c}\text { Stability to } \\
\text { hydrolysis } \\
\text { (min:sec) }\end{array}$ \\
\hline $\mathbf{2}$ & 33.5 & 6.0 & 23 & 120 & 23.48 & 180 & 37.44 \\
$\mathbf{3}$ & 32.0 & 7.0 & 20 & 115 & 39.05 & 160 & 40.12 \\
$\mathbf{4 a}$ & 35.5 & 8.5 & 24 & 135 & 42.67 & 175 & $38: 40$ \\
$\mathbf{4 b}$ & 36.0 & 8.0 & 19 & 120 & 38.09 & 185 & $34: 50$ \\
$\mathbf{5 a}$ & 34.5 & 9.5 & 18 & 100 & 56.02 & 190 & $41: 20$ \\
$\mathbf{5 b}$ & 36.0 & 8.5 & 23 & 115 & 47.06 & 215 & $37: 29$ \\
$\mathbf{5 c}$ & 35.0 & 8.6 & 22 & 95 & 49.03 & 180 & $35: 09$ \\
$\mathbf{6 a}$ & 32.0 & 10.2 & 19 & 115 & 40.01 & 215 & $37: 06$ \\
$\mathbf{6 b}$ & 33.5 & 11.5 & 24 & 117 & 37.06 & 215 & $40: 07$ \\
$\mathbf{6 c}$ & 32.5 & 9.5 & 22 & 105 & 38.00 & 205 & $36: 02$ \\
$\mathbf{7}$ & 31.5 & 10.5 & 17 & 125 & 37.04 & 210 & $48: 00$ \\
$\mathbf{8}$ & 32.5 & 11.7 & 20 & 135 & 35.09 & 205 & $44: 07$ \\
$\mathbf{9}$ & 37.0 & 8.4 & 25 & 118 & 61.02 & 165 & $35: 08$ \\
$\mathbf{1 0}$ & 36.0 & 9.3 & 18 & 105 & 57.00 & 184 & $42: 08$ \\
$\mathbf{1 1}$ & 35.5 & 10.4 & 22 & 120 & 62.01 & 179 & $45: 08$ \\
$\mathbf{1 2}$ & 32.5 & 9.4 & 18 & 104 & 46.02 & 189 & $36: 08$ \\
$\mathbf{1 3}$ & 33.0 & 8.0 & 17 & 120 & 53.01 & 210 & $35: 08$ \\
$\mathbf{1 4}$ & 35.0 & 9.4 & 19 & 110 & 49.02 & 215 & $39: 08$ \\
\hline
\end{tabular}

Error of measurements was:

Surface and interfacial tensions $= \pm 0.1 \mathrm{dynes} / \mathrm{cm}$.

Kraft point $\quad= \pm 1{ }^{\circ} \mathrm{C}$

Foam height $\quad= \pm 2 \mathrm{~mm}$

Wetting time $\quad= \pm 1 \mathrm{sec}$

Emulsion stability $\quad= \pm 1 \mathrm{~min}$ 
of measurements of individual compounds indicated such activity, they showed lower values for surface tension and interfacial tension. It was found that the decrease of values of surface and interfacial tension might be due to the electrostatic repulsion between the ionized molecules.

3.3.2. Kraft point $\left(\mathrm{T}_{\mathrm{kp}}\right)$. The Kraft point of the prepared anionic surfactants was measured at the temperature where $1 \%$ dispersion becomes clear under gradual heating. All the synthesized products are freely soluble in water. In general, $\mathrm{T}_{\mathrm{kp}}$ measurements proved that, the higher the molecular weight, the higher $\mathrm{T}_{\mathrm{kp}}$. Yet, in some cases, this fact may fail due to the presence of retarding groups in the same of molecule. So, in the case of compounds 5a-c, 6a-c, 7a-c and 12 the aryl group may increase $\mathrm{T}_{\mathrm{kp}}$ compared to the (-SH) group which causes a decrease.

3.3.3. Wetting time. The products were therefore very effective as wetting agents in distilled water. So, It is hoped that they will find a wide range of applications in the textile industry. The wetting times of the tested compounds were determined by measuring the sinking time in seconds of a gray cotton skin in the surfactant solution. The results show that the products were very effective as wetting agents in distilled water solutions.

3.3.4. Foaming height. The values of the foaming height were investigated for prepared compounds and the results revealed that the new compounds yield low foam. The low foaming power compounds have applications in the dyeing and auxiliary industries.

3.3.5. Emulsion stability. Studies are still being carried out on the utilization of surfactants in emulsion formulation, which is of immense importance to technological development. Emulsification is one of the most important properties of surfactants. All the prepared surfactants are good emulsifying agents. They could be useful in dye baths in the textile industry and as emulsion paints.

3.3.6. Stability towards hydrolysis. The results observed that the prepared compounds are moderately stable in a basic medium. Also, anionic surfactants containing heterocyclic moieties recorded high stability.

\subsection{Biodegradability}

The results showed that, biodegradability decreased with increasing molecular wieght of the compound. This indicated that, the more bulky the molecule was, the lower the biodegradability of the surfactants. Also, anionic surfactants containing heterocyclic moiety served the double function of surface active agent and antibacterial activity.

\section{CONCLUSIONS}

From the previous results, it may be concluded that all the prepared anionic surfactants have good emulsifiers in a non-edible medium such as insecticides or pesticides.

Table III

Biodegradability of the prepared compounds

\begin{tabular}{|c|c|c|c|c|c|c|c|}
\hline $\begin{array}{c}\text { Compd } \\
\text { No. }\end{array}$ & $\begin{array}{c}1^{\text {st }} \\
\text { day }\end{array}$ & $\begin{array}{l}2^{\text {nd }} \\
\text { day }\end{array}$ & $\begin{array}{l}3^{\text {rd }} \\
\text { day }\end{array}$ & $\begin{array}{l}4^{\text {th }} \\
\text { day }\end{array}$ & $\begin{array}{l}5^{\text {th }} \\
\text { day }\end{array}$ & $\begin{array}{l}6^{\text {th }} \\
\text { day }\end{array}$ & $\begin{array}{l}7^{\text {th }} \\
\text { day }\end{array}$ \\
\hline 2 & 42 & 46 & 55 & 69 & 84 & 92 & - \\
\hline 3 & 45 & 54 & 67 & 77 & 85 & 94 & - \\
\hline $4 a$ & 41 & 46 & 62 & 69 & 91 & - & - \\
\hline $4 b$ & 43 & 52 & 64 & 75 & 92 & 96 & - \\
\hline $5 a$ & 38 & 46 & 58 & 69 & 82 & 95 & - \\
\hline $5 b$ & 42 & 55 & 66 & 77 & 89 & 96 & - \\
\hline $5 c$ & 41 & 53 & 60 & 71 & 83 & 91 & - \\
\hline $6 a$ & 39 & 48 & 59 & 68 & 77 & 89 & - \\
\hline $6 b$ & 36 & 44 & 55 & 67 & 76 & 88 & 92 \\
\hline $6 c$ & 39 & 45 & 57 & 65 & 73 & 86 & 94 \\
\hline 7 & 36 & 46 & 59 & 70 & 89 & 96 & - \\
\hline 8 & 38 & 49 & 61 & 74 & 88 & 95 & 98 \\
\hline 9 & 46 & 55 & 67 & 78 & 88 & 95 & - \\
\hline 10 & 41 & 50 & 65 & 76 & 85 & 93 & - \\
\hline 11 & 46 & 55 & 67 & 78 & 88 & 95 & - \\
\hline 12 & 42 & 53 & 65 & 74 & 87 & 96 & - \\
\hline 13 & 45 & 58 & 71 & 83 & 94 & - & - \\
\hline 14 & 44 & 56 & 68 & 79 & 89 & 95 & - \\
\hline
\end{tabular}

Error of calculations was: Biodegradation rate $= \pm 0.5 \%$ 


\section{AKNOWLDGMENTS}

Origin of cultures: Botany Department, Faculty of Science, Benha University, Egypt.

\section{BIBLOGRAPHY}

Amin M. S., Eissa A. M. F., Shaaban A. F., El-Sawy A. A., El-Sayed R. 2004. New heterocycles having a double characters as antimicrobial and surface active agents. Part 1: Nonionic compounds from fatty acid isothiocyanate. Grasas Aceites. 55, 370-377.

Amin M. S., Eissa A. M. F., Shaaban A. F., El-Sawy A. A., El-Sayed R. 2003. New heterocycles having a double characters; as antimicrobial and surface active agents. Part 2: Anionic compounds from fatty acid isothiocyanate. J. Chemistry: An Indian Journal, 1, 313-319.

Boshra M. A., Ferwanah A. S., Awadallah A. M., El-Halabi N. M. 2002. Reaction of nitrilimines with ketoalkoxycarbonyl-hydrazones: Synthesis of substituted 1,2,4-triazoles and heterocyclic spiro compounds. Asian J. Chem. 14, 1235-1240.

Cansiz A., Koparir M., Demirdag A. 2004. Synthesis of some new 4,5-substituted-4H-1,2,4-triazole-3-thiol derivatives. Molecules 9, 204-212.

Eissa A. M. F. 2002. Anionic surface active agents from fatty acid hydrazides containing heterocyclic moiety. J. Olaj, Szappan, Kozmetika, 51, 155-161.

Eissa A. M. F., Ahmed M. H. M. 2003. Nonionic surface active agents containing heterocyclic moiety. J. Olaj, Szappan, Kozmetika 52,11-17.

El-Sayed R., Wasfy A. A. F., Aly A. A. 2005. Synthysis of novel heterocycles with antimicrobial and surface active agents. J. Heterocyclic Chem. 42, 1-6.

El-Sukkary M. A., El-Sawy A. A., El-Dib. 1987. Synthetic detergents from crude rice bran oil. Hung. J. Industrial Chem. 15, 317-320.

Eter E. T., Richard R. E., Darid. A. 1974. Biodegradable surfactants derived from corn starch. J. Am. Oil Chem. Soc. 51, 486 - 494.

Findly A. 1963. Practical Physical Chemistry. $6^{\text {th }}$ Ed. Longmans, London. p. 1040.

Hovsepian T. R., Dilanian E. R., Engoyan A. P., MelikOhanjaian R. G. 2004. Synthesis of substituted 1,2,4triazoles and 1,3,4-thiadiazole. J. Chem. Heterocyclic Compounds 40 1194-1198.

Katica C., Vesna D., Vlado K., Dora G. M., Aleksandra B. 2001. Synthesis, antibacterial and antifungal activity of 4-substituted-5-aryl-1,2,4-triazoles. Molecules 6, 815-824.
Kumar P. S., Nagoji K. E. V., Ravikum B.V.V. 2003. Synthesis of 3-ethoxycarbonyl-5-phenyl-1- $p$-tolyl1,2,4-triazolo[3,4,-c]1,2,4-triazole. Asian J. Chem. 15, 515-518.

Li-Xue Z., An-Jian Z., Xian-Xin C., Xin-Xiang L., XiangYun N., Dong-Yung C., Zhang Z. 2002. Synthesis and biological activity of 3-(2-furanyl)-6-aryl-1,2,4triazolo[3,4-b]-1,3,4-thiadiazoles. Molecules 7, 681689.

Masuyama A., Akiyama K., Okahara M. 1987. Surface active hydroxamic acid. II. Comparison of surface properties of hydroxamic acids with ketones and methyl esters with similar hydrophilic and lipophilic structure. J. Am. Oil. Chem. Soc. 64, 1040-1043.

Oganisyan A. Sh., Noravyan A. S., Grigoryan M. Zh. 2004. Condensed pyrido-pyrimidines. 7. Synthesis of condensed triazolo[4,3-c]- and tetrazolo[1,5-] pyrimidnes. Chem. Heterocyclic Compounds 40, 75-78.

Somaya A. R., Eissa A. M. F., Nadia. A., Ahmad, M. N. 1998. Synthesis and characterization of some peptides having surface activity using polyethylene glycol. J. Pharm. Sci. 7, 27-32.

Stankovsk S., Spirkov K. 2000. Reaction of imidoyl and amidinoyl isothiocyanates with some $\mathrm{C}$-acid salts. Chem. Papers 54, 36-38.

Takeshi H. 1970. Studies of ester containing surfactant: Preparation and properties of sodium sulphalkanoates. Bull. Chem. Soc. 43, 2236-2239.

Tayseer A. A., Manal A. D., Hamdi M. H. 2002. A novel synthesis of 1, 2,4-triazolopteridines. Molecules 7, 494-500.

Walid M. F., Pazdera P. 2002. Synthesis of heterocyclic skeletons by the reaction of N1-(2-cyanophenyl)benzimidoyl chloride with thioamides. Molecules 7 , 96-103

Wasfy A. A. F. 2003. Fused heterocycles. Part 1. Synthesis of some annelated 1,2,4-triazole system from [4-(1H-benzimidazol-2-yl)-phthalazin-1yl)hydrazine. J. Chem. Res. 8, 457-458.

Wiel J. K., Smith F. D., Stirton A. J., Bistine R. G. Jr. 1963. Long chain alkanesulphonates and 1-hydroxy-2alkanesulphonates: Structure and property relations. J. Am. Oil. Chem. Soc. 40, 538-541.

Xin-Ping H., Heng-Shan D., Peng-Fei X., Zhang Z., Qin W., Yan-Ni G. 2000. Heterocyclic systems containing bridged nitrogen atoms synthesis and antibacterial activity of 3-(2-phenylquinolin-4-yl)/3-(1-p-chlorophenyl5-methyl-1,2,3-triazol-4-yl)-s-triazolo[3,4-b]-1,3,4thiadiazine derivatives, J. Chinese Chem. Soc. 47, $1115-1119$

Recibido: Marzo 2005 Aceptado: Noviembre 2005 